\title{
To Assess the Level of Knowledge among Novice Nurses Regarding Cardiac Surgery Advance Life Support in Tertiary Care Hospital Karachi
}

\author{
Awais Jamil $^{1 *}$, Dr. Hasanat Sharif ${ }^{2}$, Mr. Jagdesh Kumar ${ }^{3}$, Dr. Muhammad Hayat ${ }^{4}$, Ms. Shaista Meghani ${ }^{5}$
}

${ }^{1}$ Clinical Nurse Manager, ${ }^{2}$ Professor, ${ }^{3}$ Head Nurse, ${ }^{4}$ Assistant Professor, ${ }^{5}$ Clinical Nurse Specialist, Tertiary Care Hospital Karachi, Pakistan

DOI: $\underline{10.36348 / \text { sjnhc.2019.v02i12.012 }}$

| Received: 16.12.2019| Accepted: 26.12.2019 | Published: 30.12.2019

*Corresponding author: Awais Jamil

\section{Abstract}

Background: Every year, over 250,000 patients have cardiac surgery and the incidence of cardiac arrest. When treated quickly, $17 \%$ to $79 \%$ of patients who experience cardiac arrest after cardiac surgery survive to discharge. Many of health care providers found this was disorganised and they would have performed better if they had well defined roles. Objectives: The aim of the study is to assess the level of knowledge among novice nurses regarding cardiac advance life support surgery (CALS) in cardiac surgery intensive care unit (CICU) and Cardio pulmonary department (D1 department) of Aga khan university hospital. Material and Methods: The descriptive cross-sectional study done. The 15 nurses were selected as sample to assess knowledge. Results: Novice nurses were not aware about (CALS). Moreover we developed module for nurses for future education and awareness about CALS the later study will help to assess the efficacy of CALS module. Conclusion: The study helps to make module of CALS for nurses taking care of patient underwent open heart surgery.

Keywords: Cardiac advance life support surgery course (CALS), Cardiac Surgery Intensive Care unit (CICU), cardiopulmonary department (D1 Ward), Advance cardiac life support Course (ACLS), Ventricular Tachycardia (VT), Ventricular Fibrillation (VF).

Copyright @ 2019: This is an open-access article distributed under the terms of the Creative Commons Attribution license which permits unrestricted use, distribution, and reproduction in any medium for non-commercial use (NonCommercial, or CC-BY-NC) provided the original author and source are credited.

\section{BACKGROUND}

Every year, over 250,000 patients have cardiac surgery and the incidence of cardiac arrest after cardiac surgery is around $0.7-2.9 \%$ [1] and has reduced in recent years. The cardiac arrest in post cardiac surgery patients may be due to reversible causes like ventricular fibrillation, major bleeding, cardiac tamponade, tension pneumothorax, and surgery specific complications.

When treated quickly, $17 \%$ to $79 \%$ of patients who experience cardiac arrest after cardiac surgery survive to discharge [2]. After cardiac surgery common complications are arrhythmias which contributes major source of morbidity and mortality. Atrial tachyarrhythmia's are the most common postoperative heart rhythm disorder. Ventricular arrhythmias and Brady arrhythmias are less frequent [3].

The literature was obtained through several electronic databases such as Google scholar, pub med, American association of critical care nurses, research gate, and the period covered in the search extended from 2006 to 2017. Keywords of cardiac AND surgery AND resuscitation was searched and it gave 102 hits and 5 of them were found relevant in terms of title abstract and other articles were found from the different sources total 11 articles were considered relevant to the initial search.

The clinical project placement area for my project was Cardiac Surgery Intensive Care Unit (CICU) and Cardio-pulmonary department (D1) from service line one. The service line one is the largest service line in Aga Khan University Hospital (AKUH) and it consists of Cardiology, Cardiothoracic Surgery (CTS) and Pulmonology departments. The targeted population of this population was the nurses of CICU and D1 who are working with cardiac surgery patients.

\section{Significance of the Study}

In 2003, in a hospital of United Kingdom, after four hours of cardiac surgery a patient had cardiac arrest and his chest was reopened three times and the patient was re-graft on his ICU bed. Many of health care providers found this was disorganised and they would have performed better if they had well defined roles. In response to this Cardiac Surgery Advance Life Support was developed, which was accepted as the European Association of Cardiothoracic Surgery's official 
protocol and has been accepted by the European Resuscitation Council (ERC) and Society of Thoracic Surgeons (STS).

The aim of the study is to critically appraise the literature review in views of current theoretical literature review which justifies the need for the present study. The review of literature is divided into three parts. The first section present the cardiac surgery advanced life support course was designed with a sequence of protocols to manage cardiac arrest patient after cardiac surgery. The second section shares the effect of this course on the management cardiothoracic surgery patients who develop cardiac arrest after cardiac surgery, while the third section will discuss the outcomes of the Cardiac Surgery Advance Life support Course (CALS).

The final section will give the limitation and gap in the available literature. It's a three days course designed by European council in this course they designed series of protocol to manage cardiac surgery patient in an arrest situation.

\section{MATERIAL AND METHODS}

The cross sectional study designed was selected for identifies the knowledge of nurses of CICU and D1 the need assessment was done from nurses. The CALS module prepares with the help of cardiothoracic surgeon and the module adopted from CALS e-learning web site. The nurses who attain the sessions about CALS were selected. The multiple choice questionnaire was developed with the help of co-preceptor and course faculty, considering the important components of CALS. The participants were selected on the voluntary bases and asked to fill the questionnaire with written informed consent. Total 15 nurses were selected to complete the questionnaire. Data was analyzed by using SPSS 20. To address the result of the need assessment three main categories (knowledge about CALS, Knowledge about rhythms, and knowledge about arrhythmias) were developed. To assess the level of knowledge each question was analyzed as correct, incorrect, and don't know.

\section{RESULTS}

\section{The below graph shows the $p$ CALS knowledge assessment of nurses}

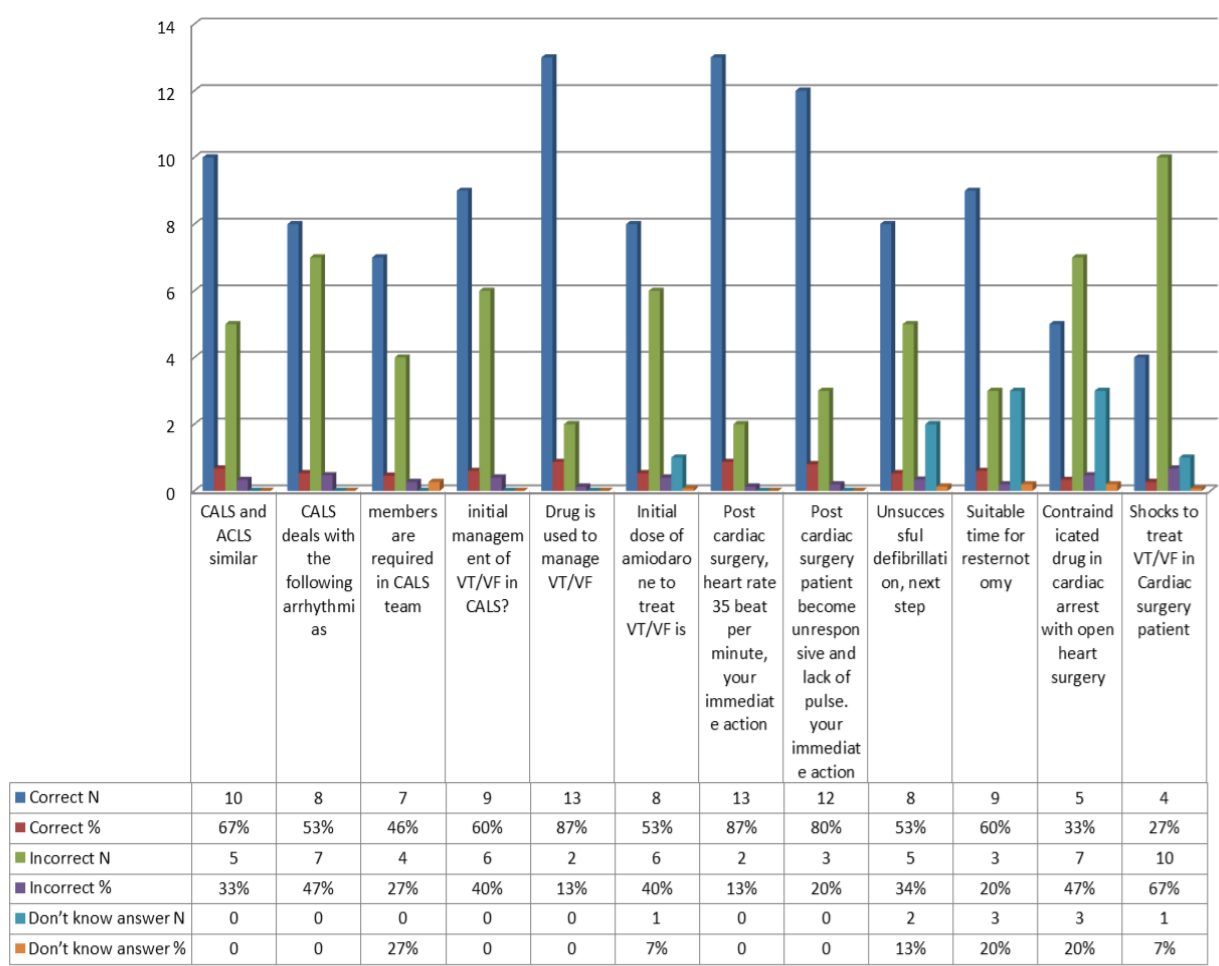

Percentage of CALS knowledge obtained in each item of knowledge

\section{DISCUSSION}

The result of the need assessment revealed that there is a need to develop a teaching module for nurses which includes all the important components about cardiac advance life support surgical. Different articles were searched from different data bases like PubMed,
CINAHIL and studied. Therefore, teaching module for the nurses who are working with cardiac surgery patients will developing after discussion with my preceptor. Furthermore, the nurses who are working with cardiac surgery patients a flyer will made according to the CALS guidelines. 
Furthermore, the expert review was taken from the preceptor and course faculty simultaneously. In the next step of the feedback was incorporated and again discus with the clinical preceptor and the module was finalized. In the last step, preceptor advised me to implement the module once the staffs of cardiac surgery get training from the experts.

\section{CONCLUSION}

The evaluation of the teaching module was not assessed, as the staff of cardiac surgery attained the orientation class about CALS and they will be trained from experts in future. As per advise of the preceptor the develop module will be implemented after the training of staff of cardiac surgery. Therefore the module was evaluated by the preceptor regarding the important concept and detailed content was covered in the module.

\section{LIMITATION}

The limitation of the project was the failure of implementing the teaching module for the health care providers of cardiac surgery due to the lack of master trainers in the hospital. However, a comprehensive teaching module was developed successfully for the staff of cardiac surgery to enhance their knowledge about CALS. Moreover, no budget was allocated for this project therefore I had to spend some money on photocopies of assessment test and consent form.

The strength of this project was its need for the staff of cardiac surgery, which will be helpful to understand the concept of CALS for long term and ultimately improve the survivor rate of cardiac surgery patients in cardiac arrest. The chosen topic was also related to my preceptor, because this topic was introduced by him. So the topic was his interest and a detailed and valuable feedback was received from him. Moreover, this module will be utilized for novice staff of cardiac surgery. The weakness of the project was that few novice nurses did not get the opportunity to attend any training session. But, they can able to understand the basic concept of CALS by reading the module.

\section{RECOMMENDATION}

- The CNIs of the CICU and D1 departments should implement the teaching module in their in service education sessions.

- The module should be kept in the crash cart folder.

\section{REFERENCES}

1. Mackay, J. H., Powell, S. J., Osgathorp, J., \& Rozario, C. J. (2002). Six-year prospective audit of chest reopening after cardiac arrest. European journal of cardio-thoracic surgery, 22(3):421-425.

2. Herrmann, C. (2014). Cardiac Advanced Life Support-Surgical Guideline: Overview and Implementation. AACN advanced critical care, 25(2):123-129.

3. Herzog, L., \& Lynch, C. (2007). Arrhythmias accompanying cardiac surgery. Clinical cardiac electrophysiology, 3:231-58. 